\title{
Visceral hypersensitivity is associated with GI symptom severity in functional GI disorders: consistent findings from five different patient cohorts
}

\author{
Magnus Simrén, ${ }^{1,2}$ Hans Törnblom, ${ }^{1}$ Olafur S Palsson, ${ }^{2}$ Miranda A L van Tilburg, ${ }^{2}$ \\ Lukas Van Oudenhove, ${ }^{3}$ Jan Tack, ${ }^{3}$ William E Whitehead ${ }^{2}$
}

Additional material is published online only. To view please visit the journal online (http://dx.doi.org/10.1136/ gutjnl-2016-312361).

Department of Internal Medicine \& Clinical Nutrition, Institute of Medicine, Sahlgrenska Academy, University of Gothenburg, Gothenburg, Sweden ${ }^{2}$ Center for Functional Gastrointestinal and Motility Disorders, University of North Carolina at Chapel Hill, Chapel Hill, North Carolina, USA ${ }^{3}$ Translational Research Center for Gastrointestinal Disorders (TARGID), University of Leuven, Leuven, Belgium

Correspondence to Professor Magnus Simrén, Department of Internal Medicine and Clinical Nutrition, Institute of Medicine, Sahlgrenska Academy, University of Gothenburg, Gothenburg 40530, Sweden;

magnus.simren@medicine.gu.se

Received 02 June 2016 Revised 16 December 2016 Accepted 01 January 2017 Published Online First 19 January 2017

\section{ABSTRACT}

Objective Our aim was to evaluate the association between visceral hypersensitivity and GI symptom severity in large cohorts of patients with functional GI disorder (FGID) and to adjust for psychological factors and general tendency to report symptoms.

Design We included five cohorts of patients with FGIDs (IBS or functional dyspepsia; $n=1144$ ), who had undergone visceral sensitivity testing using balloon distensions (gastric fundus, descending colon or rectum) and completed questionnaires to assess Gl symptom severity, non-Gl somatic symptoms, anxiety and depression. Subjects were divided into sensitivity tertiles based on pain/discomfort thresholds. Gl symptom severity was compared between sensitivity tertiles in each cohort and corrected for somatisation, and anxiety and depression.

Results In all five cohorts, Gl symptom severity increased gradually with increasing visceral sensitivity, with significant differences in GI symptom severity between the sensitivity tertiles $(p<0.0001)$, with small to medium effect sizes (partial $\eta^{2}: 0.047-0.11$ ). The differences between sensitivity tertiles remained significant in all cohorts after correction for anxiety and depression, and also after correction for non-GI somatic symptom reporting in all of the cohorts $(p<0.05)$.

Conclusions A gradual increase in Gl symptom severity with increasing GI sensitivity was demonstrated in IBS and functional dyspepsia, which was consistent across several large patient groups from different countries, different methods to assess sensitivity and assessments in different parts of the $\mathrm{Gl}$ tract. This association was independent of tendency to report symptoms or anxiety/ depression comorbidity. These findings confirm that visceral hypersensitivity is a contributor to $\mathrm{Gl}$ symptom generation in FGIDs.

\section{INTRODUCTION}

Functional GI disorders (FGIDs) are characterised by chronic and/or recurrent GI symptoms that are not explained by objective findings on standard clinical tests, such as endoscopy, radiology or blood tests. These disorders are defined by symptombased diagnostic criteria, the most recent being the Rome IV criteria. ${ }^{1} 2$ Two of the most common FGIDs are functional dyspepsia (FD) and IBS, affecting up to $25 \%$ of the general population. ${ }^{3}$
Significance of this study

What is already known on this subject?

- Visceral hypersensitivity is present in a subset of patients with functional GI disorders (FGIDs) and is considered to be one of the key pathophysiological factors in these disorders.

- Some studies have demonstrated an association between visceral sensitivity and Gl symptom severity in FGIDs, but negative studies exist.

- Psychological comorbidity and non-GI symptoms are common in patients with FGIDs and have been suggested to mediate the association between visceral sensitivity and GI symptom severity.

\section{What are the new findings?}

- A gradual increase in Gl symptom severity with increasing $\mathrm{Gl}$ sensitivity was demonstrated in five large cohorts of patients with IBS and functional dyspepsia, two of the most common FGIDs.

- This association was independent of tendency to report symptoms or anxiety/depression comorbidity.

\section{How might it impact on clinical practice in the foreseeable future?}

- Our findings suggest that visceral hypersensitivity is an important contributor to symptom generation in IBS and functional dyspepsia, and also a relevant treatment target.

Despite being very common and extensively studied, the pathophysiology of FGIDs is still incompletely understood and considered to be complex and multifactorial. Abnormal brain-gut interactions, ${ }^{5}$ psychological factors, ${ }^{5} 6$ altered GI motor function ${ }^{8}$ and visceral hypersensitivity ${ }^{7} 8$ are considered to be of relevance for FGIDs in general, but also other factors such as bacteria in the GI tract, ${ }^{9}{ }^{10}$ immune function, ${ }^{11}$ gut permeability $^{12}$ and nutrients ${ }^{13}$ have been implicated in the complex pathophysiology of FGIDs.

Abdominal pain is a dominant symptom in several FGIDs, including FD and IBS. One of the 
plausible mechanisms explaining pain and other symptoms in FGIDs is an increased sensitivity to different stimuli within the GI tract, that is, visceral hypersensitivity. This is most often studied using isobaric balloon distensions in different parts of the GI tract and quantified as pressure thresholds for discomfort and/or pain (ie, the intra-balloon pressure at which subjects first report discomfort or pain). The relevance of the concept of visceral hypersensitivity for FGIDs was first described in the early 1970s by James Ritchie, who demonstrated that patients with IBS were more sensitive to balloon distensions in the sigmoid colon than healthy controls. ${ }^{14}$ Since then numerous studies have verified the presence of visceral hypersensitivity in IBS, ${ }^{15-17}$ and also in other FGIDs such as FD $^{18}$ and functional chest pain. ${ }^{19}{ }^{20}$ However, not all patients with FGIDs demonstrate visceral hypersensitivity, and in large-scale studies approximately one-third of patients with FGIDs seem to demonstrate visceral hypersensitivity based on normal values in healthy indivi-

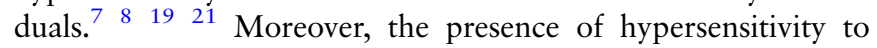
balloon distension provides no information about the underlying mechanism and its anatomical location since peripheral as well as central factors may be responsible for visceral hypersensitivity. $^{22}$

Even though it may seem logical that severity of abdominal pain and other symptoms in FGIDs should be related to visceral hypersensitivity, the existing evidence supporting such an association is moderate at best. There are indeed studies that have demonstrated an association between hypersensitivity in different parts of the GI tract and the severity of symptoms in IBS $^{15} 16{ }^{21}$ and $\mathrm{FD},{ }^{18}$ but there are also studies showing no or very weak associations between hypersensitivity and symptom reports. ${ }^{23} 24$ This divergence may relate to differences in sample size, in the protocol used to study sensory function, in questionnaires used to determine symptom severity, in patient selection and other study-related or patient-related factors. Moreover, the relevance of the visceral hypersensitivity concept for symptom generation in FGIDs, and the suggestion that it constitutes a biological marker in IBS, ${ }^{15}$ has been questioned. ${ }^{25}$ Instead, it has been proposed that visceral hypersensitivity measured by balloon distensions in the GI tract mainly reflects psychological distress or an increased tendency to report symptoms overall, respectively, rather than reflecting true neurosensory sensitivity. $^{26} 27$ In line with this, thresholds to balloon distention increase when studies are repeated over time, even if symptoms do not improve, ${ }^{28}$ and it has been suggested that selective attention to GI sensations and disease attribution may account for increased pain sensitivity in patients with FGIDs. ${ }^{25}$

Therefore, based on these existing controversies in the literature on visceral hypersensitivity in FGIDs, our aim in the present study was to test the hypothesis that visceral hypersensitivity is indeed associated with GI symptom severity and that this association is not solely explained by psychological distress or a general tendency to report symptoms. To achieve this, we evaluated the association between visceral hypersensitivity and reported severity of GI symptoms in large cohorts of patient with FGIDs, with hypersensitivity being measured by use of different protocols and in different parts of the GI tract. Furthermore, the association between visceral hypersensitivity and the severity of GI symptoms was adjusted for psychological distress and general tendency to report symptoms, respectively.

\section{METHODS}

\section{Subjects}

We included patients from five large cohorts of patients with IBS and FD, respectively, who were enrolled in studies to evaluate the pathophysiology of FGIDs. Specifically, we studied two IBS cohorts from the USA $(n=243$ and 159), two IBS cohorts from Sweden $(n=353$ and 147) and one FD cohort from Belgium $(n=244)$. The patients included in the analyses in this study underwent assessment of visceral sensitivity in the rectum, colon or gastric fundus, and completed self-report questionnaires to assess GI symptom severity, psychological distress and somatisation/somatic symptom severity (for details, see below). The inclusion criteria in the five cohorts were similar in that the patients had to fulfil the diagnostic criteria for IBS and FD at the time of inclusion, which means the Rome II criteria until 2006, ${ }^{29}{ }^{30}$ and the Rome III criteria from 2006 and onwards. $^{31} 32$ The two US IBS cohorts were evaluated in 20032007 and 2008-2013, respectively, the Swedish IBS cohorts in 2002-2010 and 2010-2014, and the Belgian FD cohort in 2002-2009. The patients were included in the studies after being referred from their primary or secondary care physicians for assessment of their FGID, through self-referral or after responding to advertisements about participation in studies about the pathophysiology of FGIDs. The FGID diagnosis was confirmed by an experienced gastroenterologist before inclusion in the study, and the absence of exclusion criteria, which were similar across studies (other GI or non-GI condition that could explain the symptoms, presence of other severe disease, predominant severe psychiatric disease, alcohol or substance abuse), was confirmed. Additional tests to rule out other GI diagnoses were performed when considered appropriate by the gastroenterologist. All patients gave verbal and written informed consent before any study-related procedures were undertaken. A proportion of the subjects included in this study have been included in previous publications using these cohorts, but not including the analyses performed in this study. ${ }^{8}$ 33-37

\section{Barostat protocols}

All patients in the five cohorts underwent a study to assess visceral sensitivity to balloon distensions using an electronic barostat (US and Swedish studies: Dual Drive Barostat, Distender Series II; G\&J Electronics, Toronto, Ontario, Canada; Belgian study: Synectics Visceral Stimulator; Synectics, Stockholm, Sweden). The details of the protocols used can be found elsewhere, ${ }^{8} 18 \quad 34 \quad 38$ but are also described in the online supplementary methods and summarised in figure 1 . In the two US IBS cohorts, the same protocol was used; phasic distensions, $30 \mathrm{~s}$ distensions, $30 \mathrm{~s}$ rest at the operating pressure, $2 \mathrm{~mm} \mathrm{Hg}$ increments, but in cohort 1 the investigation was performed in the descending colon and in cohort 2 in the rectum (figure 1A). Both Swedish IBS cohorts underwent rectal barostat studies, but with different protocols; cohort 1: phasic distensions, $30 \mathrm{~s}$ distensions, $30 \mathrm{~s}$ rest at the operating pressure, $5 \mathrm{~mm} \mathrm{Hg}$ increments (figure $1 \mathrm{~B}$ ); cohort 2 : ramp inflation starting from 0 to $4 \mathrm{~mm} \mathrm{Hg}$ increments every minute (figure 1C). The Belgian FD cohort underwent a gastric barostat test with the balloon in the gastric fundus; ramp inflation starting from operating pressure, $2 \mathrm{~mm} \mathrm{Hg}$ increments every two minutes (figure 1D).

\section{Questionnaires}

As part of the pathophysiological studies, all patients completed questionnaires to assess GI symptom severity, somatic (non-GI) symptoms, anxiety and depression. Details about these questionnaires can be found in the online supplementary methods.

\section{Gl symptom severity}

Three different questionnaires were used to assess GI symptoms in the five patient cohorts. IBS Severity Scoring System 

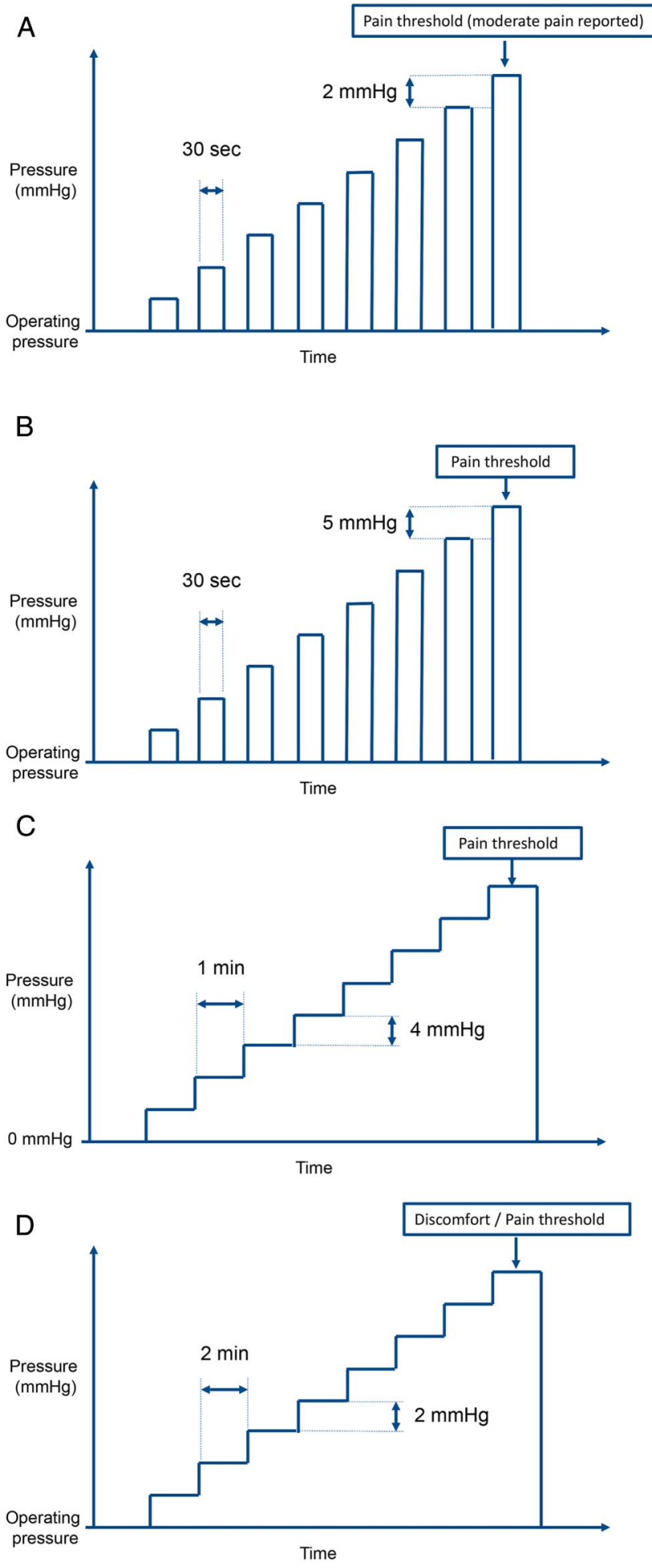

Figure 1 Schematic drawings of the barostat protocols used in both US IBS cohorts (A), in the Swedish IBS cohorts 1 (B) and 2 (C), and in the Belgian functional dyspepsia cohort (D). For more detailed explanation, please see text.

$(I B S-S S S)^{39}$ was used by a proportion of the patients with IBS in the Swedish cohort $1(\mathrm{n}=130)$ and by all patients in Swedish cohort $2(n=147)$, and by both US cohorts $(n=243$ and 159). Gastrointestinal Symptom Rating Scale-IBS (GSRS-IBS) ${ }^{40}$ was completed by a proportion of the patients in the Swedish cohort $1(\mathrm{n}=223)$, that is, by those who did not complete IBS-SSS. The Dyspepsia Symptom Severity Scale $(D S S)^{41}$ was completed by the patients in the Belgian FD cohort $(n=242)$. For this study, only the overall GI (IBS and dyspepsia, respectively) severity scores in each of these questionnaires were used.

\section{Non-Gl somatic symptoms}

Three different questionnaires were used to assess severity of somatic symptoms or somatisation in the five patient cohorts. For the purpose of this study, which for these questionnaires was to adjust for general symptom reporting tendency, only questions measuring non-GI symptoms were used. Recent Physical Symptoms Questionnaire (RPSQ) ${ }^{42}$ was used in both US cohorts $(\mathrm{n}=243$ and 159). Patient Health Questionnaire 12 $(P H Q-12)^{43}$ was used in the Belgian FD cohort $(n=242)$, by a proportion of the patients with IBS in the Swedish cohort 1 $(n=130)$ and by all patients in Swedish cohort $2(n=147)$. The proportion of patients in the Swedish cohort 2 who did not complete PHQ-12, instead completed Symptom Checklist-90 $(S C L-90)(\mathrm{n}=223),{ }^{44}$ and for this study we only used the somatisation subscale of SCL-90 with the question about GI symptoms excluded.

\section{Anxiety and depression}

Hospital Anxiety and Depression Scale $(H A D)^{45}$ was used in the Belgian and Swedish cohorts. In the US cohorts, the anxiety and depression subscales of the Brief Symptom Inventory-18 (BSI-18) were used. $^{46}$

\section{Data analysis}

Statistical analyses were performed with the software package IBM SPSS Statistics V.22 (IBM Corporation, Armonk, New York, USA). Data are presented as mean \pm SD unless stated otherwise. In the Swedish IBS cohort 1, two different questionnaires were used to assess GI symptom severity and non-GI symptoms, respectively. In order to be able to combine these, scores were transformed to $\mathrm{z}$ scores (defining how many SDs a value is from the mean, which is set to 0 ), and thereafter these were combined into one GI symptom severity variable and one non-GI symptom severity variable. To obtain comparable scales for the overall GI symptom severity in all five cohorts, $\mathrm{z}$ scores were calculated and used when displaying the results for GI (IBS and dyspepsia) severity. Based on information from the literature with a third of patients on average demonstrating visceral hypersensitivity in FGIDs, ${ }^{7} 81921$ the subjects in the five cohorts were divided into sensitivity tertiles based on their pain (IBS studies) or discomfort thresholds (FD cohort). GI symptom severity was compared between these sensitivity tertile groups using one-way analysis of variance (ANOVA) with post hoc pairwise comparisons between all three groups using independent samples t-tests with Bonferroni correction for multiple testing, as well as one-way between-groups ANOVA with linear contrast analyses to analyse the presence of a linear trend. As a next step we evaluated if comorbid anxiety and depression, on the one hand, or general symptom reporting, on the other hand, can explain a potential association between visceral sensitivity and GI symptom severity. In order to evaluate this, two different one-way between-group analyses of covariance (ANCOVA) were performed in each of the cohorts, with anxiety and depression scores used as covariates in the first model, and non-GI symptoms scores in the second model, and with sensitivity tertiles as independent variable and GI symptom severity as the dependent variable. For the purpose of comparison with other studies in this area, we also performed correlation analyses between GI symptom severity and pain/discomfort thresholds in the five different cohorts using Pearson correlation. Moreover, correlations 
between $\mathrm{z}$ scores for pain thresholds and IBS symptom severity were performed among IBS subtypes to determine if subgroup differences could be detected among patients with IBS. Statistical significance was generally accepted at $\mathrm{p}<0.05$.

\section{RESULTS}

\section{Subjects}

In total, we included 1144 subjects who fulfilled the diagnostic criteria for a FGID according to the Rome II or III criteria depending on the time of inclusion; the primary diagnosis was FD in 242 subjects (Belgian FD cohort) and IBS in 902 patients (US IBS cohorts 1 and 2, Swedish IBS cohorts 1 and 2). More detailed information about the cohorts is displayed in tables 1 and 2. There was a female predominance in all cohorts and the mean age was similar in all five cohorts. The combined IBS-A and IBS-M subgroup (Rome II and III, respectively) was the largest subgroup in all IBS cohorts except for the Swedish IBS cohort 2 where the IBS-D group was slightly larger. When subtyped according to Rome III, ${ }^{32}$ the majority of patients with FD had overlapping epigastric pain syndrome and postprandial distress syndrome. As a reflection of the different barostat protocols and the different parts of the GI tract stimulated in the sensitivity studies, the pain/discomfort thresholds differed between the cohorts. In cohorts where the same questionnaires were used, it can be seen that IBS symptom severity tended to be somewhat higher in the Swedish patients than in US patients, and Swedish patients with IBS tended to have higher levels of anxiety and depression than the Belgian FD patients. The symptom pattern in the two US cohorts was similar.

\section{Association between visceral sensitivity and GI symptom severity}

After dividing the patients in each of the five cohorts into sensitivity tertiles based on their pain (IBS cohorts) or discomfort thresholds (FD cohort), the $\mathrm{z}$ scores for GI symptom severity were compared between the sensitivity tertiles in each cohort. In all cohorts, the GI symptom severity differed significantly between the sensitivity tertiles (ANOVA: Belgian FD cohort: F $(2,239)=13.2$, partial $\eta^{2} 0.099 ; \mathrm{p}<0.0001$; US IBS cohort $1: \mathrm{F}$ $(2,240)=5.9$, partial $\eta^{2} 0.047 ; p=0.003$; US IBS cohort $2: \mathrm{F}(2$, $156)=5.1$, partial $\eta^{2} 0.061 ; p=0.007$; Swedish IBS cohort $1: \mathrm{F}$ $(2,350)=14.0$, partial $\eta^{2} 0.074 ; \mathrm{p}<0.0001$; Swedish IBS cohort
2: $F(2,144)=8.5$, partial $\left.\eta^{2} 0.11 ; p<0.0001\right)$, with small to medium effect sizes (partial $\left.\eta^{2} 0.047-0.11\right){ }^{47}$ Liner contrast analyses demonstrated a significant linear trend with an overall pattern of gradually increasing GI symptom severity with increasing GI sensitivity in the Belgian FD cohort, the US IBS cohorts and Swedish IBS cohort $1\left(\mathrm{p}<0.0001\right.$; partial $\eta^{2} 0.043-$ 0.099), but not in the Swedish IBS cohort 2, where both the linear trend and the deviation from the linear trend was significant, indicating that the linear trend could be rejected. Post hoc comparisons with Bonferroni corrections between the sensitivity tertiles demonstrated significant differences between all the sensitivity tertiles groups in the Belgian FD cohort, differences between patients with high and low thresholds in both US IBS cohorts, and differences between Swedish patients with IBS with high thresholds and the two other sensitivity tertiles groups (figure 2). In line with these findings, statistically significant negative correlations between pain/discomfort thresholds and $\mathrm{z}$ scores of GI symptom severity were seen in all cohorts, although these were of small $(<0.30)$ magnitude (table 3$).{ }^{47}$ In line with this, a negative correlation between $\mathrm{z}$ scores for pain thresholds and IBS symptom severity in all IBS subjects combined was seen $(\mathrm{r}=-0.24 ; \mathrm{p}<0.0001)$, as well as in IBS subtypes (IBS-A/M: $\mathrm{r}=$ $-0.19 ; \mathrm{p}<0.0001 ;$ IBS-C: $\mathrm{r}=-0.23 ; \mathrm{p}=0.001$; IBS-D: $\mathrm{r}=$ $-0.27 ; \mathrm{p}<0.0001)$. No differences in $\mathrm{z}$ scores for pain thresholds or IBS symptom severity were seen between IBS subtypes (data not shown).

\section{Association between visceral sensitivity and GI symptom severity adjusted for anxiety and depression}

In table 4, the results from the ANCOVA models in each of the cohorts with sensitivity tertiles as independent variable, GI symptom severity ( $\mathrm{z}$ scores) as dependent variable and anxiety and depression as covariates are displayed. After adjusting for anxiety and depression, the significant differences between the sensitivity tertiles groups on GI symptom severity remained significant in each of the five cohorts, with small to medium effect sizes (partial $\eta^{2} 0.042-0.11$ ). ${ }^{47}$ We also noted significant relationships between depression and GI symptom severity (while controlling for visceral sensitivity) in two of the cohorts (US IBS cohort 2 and Swedish IBS cohort 2), and between anxiety and GI symptom severity (while controlling for visceral sensitivity) in one of the cohorts (Swedish IBS cohort 1).

Table 1 Demographics, disease-related and study-related information in the five cohorts

\begin{tabular}{|c|c|c|c|c|c|}
\hline & Belgian cohort $(n=242)$ & US cohort $1(n=243)$ & US cohort $2(n=159)$ & Swedish cohort $1(n=353)$ & Swedish cohort $2(n=147)$ \\
\hline Primary FGID diagnosis & Functional dyspepsia & IBS & IBS & IBS & IBS \\
\hline Age* $^{*}$ years & $39 \pm 13(16-71)$ & $34 \pm 12(18-73)$ & $37 \pm 14(18-71)$ & $38 \pm 13(19-72)$ & $34 \pm 11(19-60)$ \\
\hline Sex, $n$ & $180 \mathrm{~F} / 62 \mathrm{M}$ & $203 \mathrm{~F} / 40 \mathrm{M}$ & $126 \mathrm{~F} / 33 \mathrm{M}$ & $267 \mathrm{~F} / 86 \mathrm{M}$ & $102 \mathrm{~F} / 45 \mathrm{M}$ \\
\hline Barostat & Stomach & Colon & Rectum & Rectum & Rectum \\
\hline \multicolumn{6}{|l|}{ IBS subtype† \% } \\
\hline IBS-C & & $18 \%$ & $23 \%$ & $24 \%$ & $27 \%$ \\
\hline IBS-D & & $33 \%$ & $24 \%$ & $36 \%$ & $37 \%$ \\
\hline IBS-M/IBS- A & & $49 \%$ & $53 \%$ & $40 \%$ & $36 \%$ \\
\hline \multicolumn{6}{|l|}{ FD subtype } \\
\hline EPS & $8 \%$ & & & & \\
\hline PDS & $36 \%$ & & & & \\
\hline Overlap EPS and PDS & $47 \%$ & & & & \\
\hline Not possible to subtype & $9 \%$ & & & & \\
\hline
\end{tabular}

${ }^{*}$ Age displayed as mean $\pm S D$ and range. 
Table 2 Outcome measures used in the five cohorts (mean \pm SD)

\begin{tabular}{|c|c|c|c|c|c|}
\hline & $\begin{array}{l}\text { Belgian cohort } \\
(\mathrm{n}=242)\end{array}$ & $\begin{array}{l}\text { US cohort } 1 \\
(n=243)\end{array}$ & $\begin{array}{l}\text { US cohort } 2 \\
(n=159)\end{array}$ & $\begin{array}{l}\text { Swedish cohort } 1 \\
(n=353)\end{array}$ & $\begin{array}{l}\text { Swedish cohort } 2 \\
(n=147)\end{array}$ \\
\hline $\begin{array}{l}\text { Pain/discomfort threshold } \\
(\mathrm{mm} \mathrm{Hg})\end{array}$ & $10 \pm 4$ & $26 \pm 13$ & $16 \pm 8$ & $33 \pm 13$ & $27 \pm 8.8$ \\
\hline \multicolumn{6}{|l|}{ Gl symptoms } \\
\hline IBS-SSS & & $275 \pm 91$ & $284 \pm 92$ & $304 \pm 90$ & $315 \pm 91$ \\
\hline GSRS-IBS & & & & $3.5 \pm 0.95$ & \\
\hline DSS & $14 \pm 5$ & & & & \\
\hline \multicolumn{6}{|l|}{ Non-Gl symptoms } \\
\hline SCL-90 som & & & & $0.89 \pm 0.71$ & \\
\hline PHQ-12 & $8.9 \pm 4.2$ & & & $7.4 \pm 4.0$ & $8.5 \pm 4.1$ \\
\hline RPSQ & & $11 \pm 5.2$ & $11 \pm 5.4$ & & \\
\hline \multicolumn{6}{|l|}{ Anxiety } \\
\hline HAD & $6.2 \pm 4.1$ & & & $7.7 \pm 4.6$ & $8.5 \pm 4.6$ \\
\hline BSI-18 & & $52 \pm 11$ & $51 \pm 9.7$ & & \\
\hline \multicolumn{6}{|l|}{ Depression } \\
\hline $\mathrm{HAD}$ & $4.4 \pm 3.7$ & & & $5.1 \pm 3.7$ & $5.3 \pm 3.5$ \\
\hline BSI-18 & & $52 \pm 9.9$ & $53 \pm 11$ & & \\
\hline
\end{tabular}

The sensitivity parameters are displayed as thresholds in $\mathrm{mm} \mathrm{Hg}$ above the operating pressure for all cohorts except for Swedish cohort 2, as this distension protocol started from $0 \mathrm{~mm} \mathrm{Hg}$, whereas all the others distension protocols started from the operating pressure.

Empty cells indicate that this variable was not used in the cohort.

BSI-18, Brief Symptom Inventory-18; DSS, Dyspepsia Symptom Severity Scale; GSRS-IBS, Gastrointestinal Symptom Rating Scale-IBS; HAD, Hospital Anxiety and Depression Scale; IBS-SSS, IBS-Severity Scoring System; PHQ-12, Patient Health Questionnaire 12; RPSQ, Recent Physical Symptoms Questionnaire; SCL-90, Symptom Checklist-90; SCL-90 som, SCL-90 somatisation subscale without GI sX.

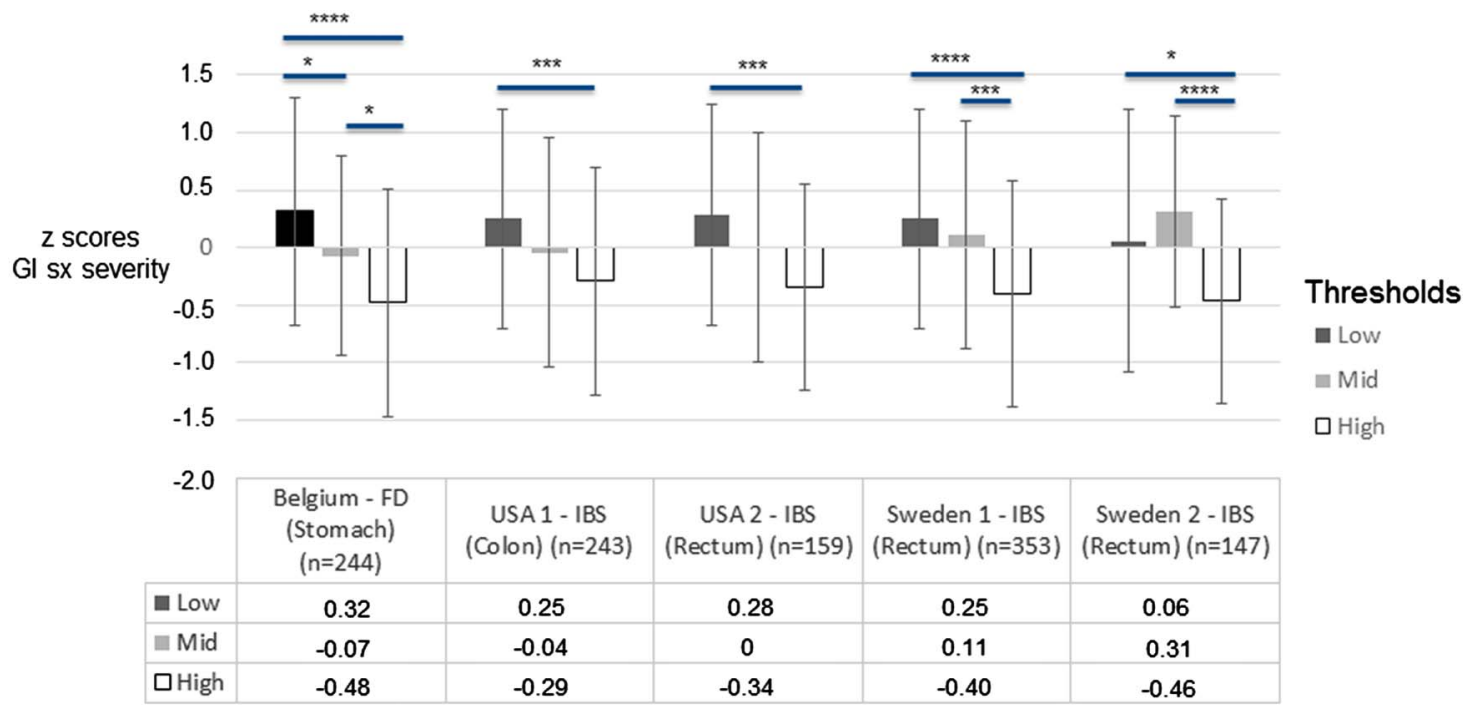

Figure $2 \mathrm{Gl}$ symptom severity (z score (SD from the mean (0) value) in the sensitivity tertiles based on pain or discomfort threshold (low, mid, high). Analysis of variance with Bonferroni corrected post hoc comparisons between the groups $\left({ }^{*} p<0.05 ;{ }^{* *} p<0.01 ;{ }^{* * *} p<0.001 ;{ }^{* * * *} p<0.0001\right)$. FD, functional dyspepsia.

\section{Association between visceral sensitivity and GI symptom severity adjusted for non-GI symptom severity}

In table 5, the results from the ANCOVA models in each of the cohorts with sensitivity tertiles as independent variable, GI symptom severity (z scores) as dependent variable and non-GI sx severity as covariate are displayed. After adjusting for non-GI symptom severity, the significant differences between the sensitivity tertiles groups on GI symptom severity remained significant in each of the five cohorts, with small to medium effect sizes (partial $\eta^{2}$ 0.039-0.073). ${ }^{47}$ We also noted significant relationships between non-GI and GI symptom severity (while controlling for visceral sensitivity) in all of the cohorts with mainly medium to large effect sizes.

\section{DISCUSSION}

In this study, we demonstrated a gradual increase in GI symptom severity with increasing visceral sensitivity in IBS and FD, which was remarkably consistent across several large patient groups from different countries, different methods to assess sensitivity and assessments of sensitivity in different parts of the GI tract. This association, although modest, was still significant after adjustment for general tendency to report symptoms or anxiety/depression comorbidity. These findings support that visceral hypersensitivity is an important contributor to symptom generation in IBS and FD.

Visceral hypersensitivity has for long been considered to play a central role in the pathophysiology of FGIDs, contributing to 
Table 3 Pearson correlation between pain/discomfort thresholds and $\mathrm{z}$ scores of $\mathrm{Gl}$ symptom severity in the five patient cohorts

\begin{tabular}{llc}
\hline & Correlation coefficient & p Value \\
\hline Belgian cohort $(n=242)$ & -0.27 & $<0.0001$ \\
US cohort 1 $(n=243)$ & -0.20 & 0.002 \\
US cohort 2 $(n=159)$ & -0.27 & 0.001 \\
Swedish cohort 1 $(n=353)$ & -0.29 & $<0.0001$ \\
Swedish cohort 2 $(n=147)$ & -0.20 & 0.02 \\
\hline
\end{tabular}

Table 4 Summary of results from one-way between-group analyses of covariance with visceral sensitivity tertiles as independent variable, GI symptom severity (z scores) as dependent variable and anxiety and depression as covariates in each of the cohorts

\begin{tabular}{llll}
\hline & Visceral sensitivity & Anxiety & Depression \\
\hline Belgian functional dyspepsia cohort & & \\
$\mathrm{F}$ & 13.3 & 0.72 & 2.9 \\
$\mathrm{p}$ Value & $<0.0001$ & 0.40 & 0.092 \\
Partial $\eta^{2}$ & 0.10 & 0.003 & 0.012 \\
$\mathrm{R}^{2}$ & 0.11 & & \\
US IBS cohort 1 & & & \\
$\mathrm{F}$ & 5.3 & 0.41 & 2.4 \\
$\mathrm{p}$ Value & 0.006 & 0.52 & 0.13 \\
Partial $\eta^{2}$ & 0.042 & 0.002 & 0.01 \\
$\mathrm{R}^{2}$ & 0.08 & & \\
US IBS cohort 2 & & & \\
$\mathrm{F}$ & 4.1 & 0.13 & 6.1 \\
$\mathrm{p}$ Value & 0.018 & 0.72 & 0.015 \\
Partial $\eta^{2}$ & 0.051 & 0.001 & 0.038 \\
$\mathrm{R}^{2}$ & 0.13 & & \\
Swedish IBS cohort 1 & & & \\
$\mathrm{F}$ & 11.5 & 6.5 & 3.5 \\
$\mathrm{p}$ Value & $<0.0001$ & 0.011 & 0.063 \\
Partial $\eta^{2}$ & 0.062 & 0.018 & 0.010 \\
$\mathrm{R}^{2}$ & 0.14 & & \\
Swedish IBS cohort 2 & & & \\
$\mathrm{F}$ & 8.3 & 0.66 & 9.5 \\
$\mathrm{p}$ Value & $<0.0001$ & 0.42 & 0.002 \\
Partial $\eta^{2}$ & 0.11 & & 0.064 \\
$\mathrm{R}^{2}$ & 0.22 & & \\
\hline
\end{tabular}

Significant differences shown in bold.

some of the key symptoms in these large groups of patients. In FD, large-scale studies have demonstrated an association with pain predominance, ${ }^{7}$ and in patients with non-cardiac chest pain, oesophageal hypersensitivity can be found in approximately a third of the patients. ${ }^{19}$ In IBS, an association with the overall severity of IBS symptoms have been found in several large studies, ${ }^{15} 162134$ and among the individual IBS symptoms the most consistent associations have been demonstrated with pain and bloating. ${ }^{16} 34$ However, there are also studies in patients with FGID where no or very weak associations between visceral hypersensitivity and severity of GI symptoms were found. ${ }^{23} 24$ Several explanations for these discrepant findings exist, such as differences in sample size, protocol used to study sensitivity, questionnaires used to determine symptom severity, patient selection and other study-related or patient-related
Table 5 Summary of results from one-way between-group analyses of covariance with visceral sensitivity tertiles as independent variable, GI symptom severity (z scores) as dependent variable and non-GI sx severity as covariate in each of the cohorts

\begin{tabular}{|c|c|c|}
\hline & Visceral sensitivity & Non-GI sx severity \\
\hline \multicolumn{3}{|c|}{ Belgian functional dyspepsia cohort } \\
\hline $\mathrm{F}$ & 9.3 & 25.9 \\
\hline p Value & $<0.0001$ & $<0.0001$ \\
\hline Partial $\eta^{2}$ & 0.073 & 0.099 \\
\hline $\mathrm{R}^{2}$ & 0.19 & \\
\hline \multicolumn{3}{|c|}{ US IBS cohort 1} \\
\hline $\mathrm{F}$ & 4.9 & 9.9 \\
\hline p Value & 0.008 & 0.002 \\
\hline Partial $\eta^{2}$ & 0.040 & 0.040 \\
\hline $\mathrm{R}^{2}$ & 0.09 & \\
\hline \multicolumn{3}{|c|}{ US IBS cohort 2} \\
\hline $\mathrm{F}$ & 3.1 & 21.2 \\
\hline p Value & 0.046 & $<0.0001$ \\
\hline Partial $\eta^{2}$ & 0.039 & 0.12 \\
\hline $\mathrm{R}^{2}$ & 0.17 & \\
\hline \multicolumn{3}{|c|}{ Swedish IBS cohort 1} \\
\hline $\mathrm{F}$ & 9.1 & 62.8 \\
\hline p Value & 0.002 & $<0.0001$ \\
\hline Partial $\eta^{2}$ & 0.047 & 0.196 \\
\hline $\mathrm{R}^{2}$ & 0.25 & \\
\hline \multicolumn{3}{|c|}{ Swedish IBS cohort 2} \\
\hline $\mathrm{F}$ & 3.9 & 23.1 \\
\hline p Value & 0.023 & $<0.0001$ \\
\hline Partial $\eta^{2}$ & 0.052 & 0.141 \\
\hline $\mathrm{R}^{2}$ & 0.23 & \\
\hline
\end{tabular}

factors. Also, the statistical approach and definitions of hypersensitivity may partly explain discrepant results.

In the present study, where we included patients with different FGIDs emanating from different countries, who were studied with different barostat protocols in different parts of the GI tract, we were able to show remarkably similar results across the cohorts. In all cohorts, and in both disorders, we found a gradual increase in GI symptom severity with increasing visceral sensitivity to balloon distention. The strength of this association between visceral sensitivity and the severity of GI symptoms was modest, although consistent and robust across cohorts, and in ANCOVA models we demonstrated small to medium effect sizes for these associations. This implicates that visceral sensitivity is indeed of relevance for GI symptom severity in FGIDs, but additional factors not measured in our studies most likely also play an important role in symptom generation.

Psychological factors, such as anxiety and depression, are of great importance for symptom severity and quality of life in patients with FGIDs, ${ }^{5} 621$ and in patients seeking healthcare for FGIDs a large proportion also fulfil diagnostic criteria for a psychiatric disorder. ${ }^{5}$ Regarding the relevance of visceral hypersensitivity for GI symptom severity, it has been proposed that this association could be explained by coexistence of psychological symptoms and reporting bias. In fact, in some studies visceral hypersensitivity was found to be associated with psychological comorbidity, but not with GI symptoms. ${ }^{24}$ Moreover, several studies also show that the psychological state of the individual affects symptom perception. ${ }^{20} 252748$ Indeed, state anxiety is inversely correlated with discomfort thresholds to balloon 
distention in patients with hypersensitive $\mathrm{FD},{ }^{49}$ and increased colonic sensitivity in IBS seems to be strongly influenced by psychological factors. $^{26}$ Therefore, one of the aims in our study was to evaluate if the association between visceral hypersensitivity and GI symptom severity in our large patient cohorts could be explained by comorbid anxiety and/or depression. We found that, across all five cohorts, the association between GI sensitivity and GI symptom severity remained significant after controlling for psychological distress. This implicates that even though psychological comorbidity undoubtedly is of importance for symptom generation in FGIDs, the association between GI symptom severity and GI sensitivity cannot be explained by coexisting psychological distress.

Another factor considered to be of importance for symptom generation in FGIDs is somatisation, or a general tendency to report several unexplained somatic symptoms. Somatisation has indeed been found to be associated with visceral hypersensitivity in FGIDs, ${ }^{26} 27$ as well as to be of relevance for GI symptom severity in patients with FGID. ${ }^{37} 50$ In war veterans with IBS, the pain sensitivity was largely explained by psychological measures, including somatic focus, ${ }^{51}$ and in a recent study we found somatisation to be associated with an exaggerated postprandial symptom response in patients with IBS. ${ }^{52}$ Moreover, in a study with the primary aim to determine whether lower visceral pain thresholds in IBS primarily reflect physiological or psychological factors, colonic sensitivity was found to be associated with a psychological tendency to report symptoms and this tendency was related to somatisation. ${ }^{26}$ Therefore, in the present study we aimed to determine whether the association between GI sensitivity and GI symptom severity could be explained by a general tendency to report symptoms, assessed with different measures of non-GI symptom severity in our cohorts. Even though our study was no exception from previous studies by showing that somatic symptom reporting or somatisation is indeed strongly associated with GI symptom severity, the association between GI sensitivity and GI symptom severity remained significant after adjustment for non-GI symptom severity, suggesting that this association cannot solely be explained by a general tendency to report symptoms.

The optimal way to show that visceral hypersensitivity is of importance for symptom generation in FGIDs is of course to show that an increased visceral pain threshold reduces GI symptoms. To date, there are few studies using this approach to support the association, but there is some limited data demonstrating parallel improvement in GI sensitivity and GI symptoms with treatment in small groups of patients with IBS. ${ }^{53}$ On the other hand, there are also studies with medications that reduce GI symptoms in IBS in the absence of a significant effect on visceral sensitivity. ${ }^{54}$ Interestingly, a recently published randomised double-blind placebo-controlled study using the histamine receptor $\mathrm{H} 1$ antagonist ebastine to treat IBS symptoms could show that IBS symptoms improved in parallel with a reduction in visceral sensitivity, supporting the relevance of visceral hypersensitivity for symptom generation in IBS. ${ }^{55}$ More treatment trials incorporating measures of visceral sensitivity and symptoms are needed to further determine the relevance of visceral hypersensitivity for GI symptom generation in various FGIDs.

There are of course limitations to our study. Including five different patient cohorts with different barostat protocols to test visceral sensitivity and different questionnaires to assess GI symptoms, psychological distress and non-GI symptoms might be viewed as a weakness. Conversely, this could also be considered a strength since the findings in the different cohorts were remarkably similar if evaluated by an appropriate statistical approach and data analysis. Both the association between GI sensitivity and symptoms, as well as the effect of psychological distress and tendency to report symptoms on this association are robust between studies. Moreover, the association between pain sensitivity and GI symptom severity was similar among IBS subtypes. Another potential limitation is that our studies were performed in specialised centres and mainly included patients who were referred from primary care to the specialised units, which makes it difficult to generalise our findings to all patients with IBS. On the other hand, increased pain sensitivity does not seem be only confined to select groups of patients with severe FGIDs, but can also be found in population-based studies. Visceral hypersensitivity has also been related to the severity of IBS in such cohorts, ${ }^{56}$ and in blood donors with dyspeptic symptoms, ${ }^{57}$ which fits well with our findings of an association between symptom severity and sensitivity. The patients in these studies were included based on Rome II $^{29}{ }^{30}$ or Rome III diagnostic criteria, ${ }^{31} 32$ and how changes in the recent Rome IV diagnostic criteria, which tend to be more strict, at least for IBS, will influence our findings cannot be assessed. ${ }^{12}$ However, it is quite likely that the vast majority of patients included in our study would also fulfil the Rome IV criteria since mild and infrequent symptoms were uncommon among our subjects.

In conclusion, visceral sensitivity is associated with the severity of GI symptoms in IBS and this association cannot be explained by a general tendency to report symptoms or by psychological distress. These findings suggest that visceral hypersensitivity is an important contributor to symptom generation in IBS and FD, and probably also a relevant treatment target.

Contributors Inclusion of patients and critical revision of manuscript: all co-authors. Statistical analyses: MS and LVO. Manuscript draft: MS. Obtained funding: MS, JT and WEW.

Funding This study was supported by NIDDK (grant R01 DK31369), the Swedish Medical Research Council (grants 13409, 21691 and 21692), AFA Insurance, unrestricted grants from Ferring Pharmaceuticals and Danone Research, and by the Faculty of Medicine, University of Gothenburg. LVO is a Research Professor funded by the KU Leuven Special Research Fund (Bijzonder Onderzoeksfonds, BOF).

Competing interests MS has received unrestricted research grants from Danone, and Ferring Pharmaceuticals, and served as a Consultant/Advisory Board member for AstraZeneca, Danone, Nestlé, Chr Hansen, Almirall, Allergan, Albireo, Glycom and Shire, and as a speaker for Tillotts, Takeda, Shire and Almirall. HT has served as Consultant/Advisory Board member for Almirall, Danone and Shire. MALvT has obtained research support from Takeda for investigator initiated study. OSP has received salary support from a research grants from Takeda Pharmaceuticals and Salix Pharmaceuticals and from a consulting agreement with Ironwood Pharmaceuticals and an educational grant provided by Takeda Pharmaceuticals, and received a speaker honorarium in an educational programme supported by Ironwood Pharmaceuticals and Takeda Pharmaceuticals. JT has given scientific advice to Almirall, AstraZeneca, Danone, Menarini, Novartis, Nycomed, Ocera, Ono pharma, Shire, SK Life Sciences, Theravance, Tranzyme, Xenoport, and Zeria Pharmaceuticals, and has been member of the Speaker bureau for: Abbott, Almirall, AlfaWasserman, AstraZeneca, Janssen, Menarini, Novartis, Nycomed, Shire, Zeria. WEW received research grants from Takeda, Ironwood, Salix, and the Rome Foundation; served as a consultant to Biomerica USA, Ono Pharmaceuticals and Ferring; and received unrestricted educational grants from Takeda and Ferring.

\section{Patient consent Obtained.}

Ethics approval Regional Ethical Review Boards in Chapel Hill, Gothenburg or Leuven.

Provenance and peer review Not commissioned; externally peer reviewed.

\section{REFERENCES}

1 Lacy BE, Mearin F, Chang L, et al. Bowel Disorders. Gastroenterology 2016;150:1393-407.e5.

2 Stanghellini V, Chan FK, Hasler WL, et al. Gastroduodenal Disorders. Gastroenterology 2016;150:1380-92.

3 Lovell RM, Ford AC. Global prevalence of and risk factors for irritable bowel syndrome: a meta-analysis. Clin Gastroenterol Hepatol 2012;10:712-21.e4. 
4 Mahadeva S, Ford AC. Clinical and epidemiological differences in functional dyspepsia between the East and the West. Neurogastroenterol Motil 2016;28:167-74.

5 Van Oudenhove L, Aziz Q. The role of psychosocial factors and psychiatric disorders in functional dyspepsia. Nat Rev Gastroenterol Hepatol 2013;10:158-67.

6 Jerndal P, Ringström G, Agerforz P, et al. Gastrointestinal-specific anxiety: an important factor for severity of GI symptoms and quality of life in IBS. Neurogastroenterol Motil 2010:22:646-e179.

7 Karamanolis G, Caenepeel P, Arts J, et al. Association of the predominant symptom with clinical characteristics and pathophysiological mechanisms in functional dyspepsia. Gastroenterology 2006;130:296-303.

8 Törnblom H, Van Oudenhove L, Tack J, et al. Interaction between preprandial and postprandial rectal sensory and motor abnormalities in IBS. Gut 2014;63: 1441-9.

9 Jeffery IB, O'Toole PW, Öhman L, et al. An irritable bowel syndrome subtype defined by species-specific alterations in faecal microbiota. Gut 2012;61:997-1006

10 Suzuki H, Moayyedi P. Helicobacter pylori infection in functional dyspepsia. Nat Rev Gastroenterol Hepatol 2013;10:168-74.

11 Keely S, Walker MM, Marks E, et al. Immune dysregulation in the functional gastrointestinal disorders. Eur J Clin Invest 2015;45:1350-9.

12 Piche T. Tight junctions and IBS-the link between epithelial permeability, low-grade inflammation, and symptom generation? Neurogastroenterol Motil 2014;26:296-302.

13 Farré R, Tack J. Food and symptom generation in functional gastrointestinal disorders: physiological aspects. Am J Gastroenterol 2013;108:698-706.

14 Ritchie J. Pain from distension of the pelvic colon by inflating a balloon in the irritable colon syndrome. Gut 1973;14:125-32.

15 Mertz $\mathrm{H}$, Naliboff $\mathrm{B}$, Munakata J, et al. Altered rectal perception is a biological marker of patients with irritable bowel syndrome. Gastroenterology 1995; 109:40-52.

16 Posserud I, Syrous A, Lindström L, et al. Altered rectal perception in irritable bowel syndrome is associated with symptom severity. Gastroenterology 2007:133:1113-23.

17 Whitehead WE, Engel BT, Schuster MM. Irritable bowel syndrome: physiological and psychological differences between diarrhea-predominant and constipation-predominant patients. Dig Dis Sci 1980;25:404-13.

18 Tack J, Caenepeel P, Fischler B, et al. Symptoms associated with hypersensitivity to gastric distention in functional dyspepsia. Gastroenterology 2001;121:526-35.

19 Nasr I, Attaluri A, Coss-Adame E, et al. Diagnostic utility of the oesophageal balloon distension test in the evaluation of oesophageal chest pain. Aliment Pharmacol Ther 2012;35:1474-81.

20 Farmer AD, Coen SJ, Kano M, et al. Psychophysiological responses to visceral and somatic pain in functional chest pain identify clinically relevant pain clusters. Neurogastroenterol Motil 2014;26:139-48.

21 van der Veek PP, Van Rood YR, Masclee AA. Symptom severity but not psychopathology predicts visceral hypersensitivity in irritable bowel syndrome. Clin Gastroenterol Hepatol 2008;6:321-8.

22 Elsenbruch S. Abdominal pain in Irritable Bowel Syndrome: a review of putative psychological, neural and neuro-immune mechanisms. Brain Behav Immun 2011:25:386-94

23 Boeckxstaens GE, Hirsch DP, Kuiken SD, et al. The proximal stomach and postprandial symptoms in functional dyspeptics. Am J Gastroenterol 2002;97:40-8.

24 Sabate JM, Veyrac M, Mion F, et al. Relationship between rectal sensitivity, symptoms intensity and quality of life in patients with irritable bowel syndrome. Aliment Pharmacol Ther 2008:28:484-90.

25 Whitehead WE, Palsson OS. Is rectal pain sensitivity a biological marker for irritable bowel syndrome: psychological influences on pain perception. Gastroenterology 1998;115:1263-71.

26 Dorn SD, Palsson OS, Thiwan SI, et al. Increased colonic pain sensitivity in irritable bowel syndrome is the result of an increased tendency to report pain rather than increased neurosensory sensitivity. Gut 2007;56:1202-9.

27 Van Oudenhove L, Vandenberghe J, Vos R, et al. Abuse history, depression, and somatization are associated with gastric sensitivity and gastric emptying in functional dyspepsia. Psychosom Med 2011;73:648-55.

28 Naliboff BD, Berman S, Suyenobu B, et al. Longitudinal change in perceptual and brain activation response to visceral stimuli in irritable bowel syndrome patients. Gastroenterology 2006;131:352-65.

29 Talley NJ, Stanghellini V, Heading RC, et al. Functional gastroduodenal disorders. Gut 1999;45(Suppl 2):II37-42.

30 Thompson WG, Longstreth GF, Drossman DA, et al. Functional bowel disorders and functional abdominal pain. Gut 1999;45(Suppl 2):॥43-7.

31 Longstreth GF, Thompson WG, Chey WD, et al. Functional bowel disorders. Gastroenterology 2006;130:1480-91.
32 Tack J, Talley NJ, Camilleri M, et al. Functional gastroduodenal disorders. Gastroenterology 2006;130:1466-79.

33 Clauwaert $\mathrm{N}$, Jones MP, Holvoet $\mathrm{L}$, et al. Associations between gastric sensorimotor function, depression, somatization, and symptom-based subgroups in functional gastroduodenal disorders: are all symptoms equal? Neurogastroenterol Motil 2012;24:1088-e565.

34 Kanazawa M, Palsson OS, Thiwan SI, et al. Contributions of pain sensitivity and colonic motility to IBS symptom severity and predominant bowel habits. Am J Gastroenterol 2008;103:2550-61.

35 Le Nevé $B$, Brazeilles $R$, Derrien $M$, et al. Lactulose challenge determines visceral sensitivity and severity of symptoms in patients with irritable bowel syndrome. Clin Gastroenterol Hepatol 2016:14:226-33.e1-3.

36 Ly HG, Weltens N, Tack J, et al. Acute anxiety and anxiety disorders are associated with impaired gastric accommodation in patients with functional dyspepsia. Clin Gastroenterol Hepatol 2015;13:1584-91.e3.

37 van Tilburg MA, Palsson OS, Whitehead WE. Which psychological factors exacerbate irritable bowel syndrome? Development of a comprehensive model. J Psychosom Res 2013;74:486-92.

38 Cremonini F, Houghton LA, Camilleri $M$, et al. Barostat testing of rectal sensation and compliance in humans: comparison of results across two centres and overall reproducibility. Neurogastroenterol Motil 2005;17:810-20.

39 Francis CY, Morris J, Whorwell PJ. The irritable bowel severity scoring system: a simple method of monitoring irritable bowel syndrome and its progress. Aliment Pharmacol Ther 1997;11:395-402.

40 Wiklund IK, Fullerton S, Hawkey CJ, et al. An irritable bowel syndrome-specific symptom questionnaire: development and validation. Scand I Gastroenterol 2003;38:947-54.

41 Cuomo R, Sarnelli G, Grasso R, et al. Functional dyspepsia symptoms, gastric emptying and satiety provocative test: analysis of relationships. Scand J Gastroentero/ 2001;36:1030-6.

42 MacLean EW, Palsson OS, Turner MJ, et al. Development and validation of new disease-specific measures of somatization and comorbidity in IBS. J Psychosom Res 2012:73:351-5

43 Spiller RC, Humes DJ, Campbell E, et al. The Patient Health Questionnaire 12 Somatic Symptom scale as a predictor of symptom severity and consulting behaviour in patients with irritable bowel syndrome and symptomatic diverticular disease. Aliment Pharmacol Ther 2010;32:811-20.

44 Derogatis LR, Rickels K, Rock AF. The SCL-90 and the MMPI: a step in the validation of a new self-report scale. Br J Psychiatry 1976;128:280-9.

45 Zigmond AS, Snaith RP. The hospital anxiety and depression scale. Acta Psychiatr Scand 1983;67:361-70.

46 Derogatis LR. BSI 18, Brief Symptom Inventory 18: administration, scoring, and procedures manual. NCS Pearson, Inc., 2000.

47 Cohen J. Statistical power analysis for the behavioral sciencies. Routledge, 1988.

48 Grinsvall C, Törnblom H, Tack J, et al. Psychological factors selectively upregulate rectal pain perception in hypersensitive patients with irritable bowel syndrome. Neurogastroenterol Motil 2015;27:1772-82.

49 Van Oudenhove L, Vandenberghe J, Geeraerts B, et al. Relationship between anxiety and gastric sensorimotor function in functional dyspepsia. Psychosom Med 2007:69:455-63.

50 Van Oudenhove L, Vandenberghe J, Geeraerts B, et al. Determinants of symptoms in functional dyspepsia: gastric sensorimotor function, psychosocial factors or somatisation? Gut 2008:57:1666-73.

51 Dunphy RC, Bridgewater L, Price DD, et al. Visceral and cutaneous hypersensitivity in Persian Gulf war veterans with chronic gastrointestinal symptoms. Pain 2003;102:79-85.

52 Van Oudenhove L, Törnblom H, Störsrud S, et al. Depression and somatization are associated with increased postprandial symptoms in patients with irritable bowel syndrome. Gastroenterology 2016;150:866-74.

53 Poitras $\mathrm{P}$, Riberdy Poitras M, Plourde V, et al. Evolution of visceral sensitivity in patients with irritable bowel syndrome. Dig Dis Sci 2002;47:914-20.

54 Tack J, Broekaert D, Fischler B, et al. A controlled crossover study of the selective serotonin reuptake inhibitor citalopram in irritable bowel syndrome. Gut 2006:55:1095-103.

55 Wouters MM, Balemans D, Van Wanrooy S, et al. Histamine Receptor H1-Mediated Sensitization of TRPV1 Mediates Visceral Hypersensitivity and Symptoms in Patients With Irritable Bowel Syndrome. Gastroenterology 2016;150:875-87.e9.

56 Stabell N, Stubhaug A, Flægstad T, et al. Increased pain sensitivity among adults reporting irritable bowel syndrome symptoms in a large population-based study. Pain 2013;154:385-92.

57 Holtmann G, Gschossmann J, Neufang-Hüber J, et al. Differences in gastric mechanosensory function after repeated ramp distensions in non-consulters with dyspepsia and healthy controls. Gut 2000;47:332-6. 